\title{
UNTERSUCHUNG DES KOLLEKTIVEN SCHUTZES SOLIDARITÄTSRECHTE
}

\section{ORIGINALER ARTIKEL}

RIBEIRO, Danilo Miranda ${ }^{1}$

RIBEIRO, Danilo Miranda. Untersuchung des kollektiven Schutzes

Solidaritätsrechte. Revista Científica Multidisciplinar Núcleo do Conhecimento. Jahr. 06, Hrsg. 12, Vol. 02, S. 152-167. Dezember 2021. ISSN: 2448-0959, Anfahrtslink: https://www.nucleodoconhecimento.com.br/gesetz/solidaritaetsrechte, DOI: 10.32749/nucleodoconhecimento.com.br/gesetz/solidaritaetsrechte

\section{ZUSAMMENFASSUNG}

Diese Arbeit zielt darauf ab, eine rechtliche Analyse über die Elemente des kollektiven Prozesses zum Schutz Solidaritätsrechte im brasilianischen Rechtssystem aufzubauen. Die Erforschung der kollektiven Bevormundung steht aufgrund ihrer gesetzmäßigen Distanzierung vor dem Problem eines schwer verständlichen hermetischen Systems. Mit dem Ziel, Platz für die Untersuchung des kollektiven Prozesses zu schaffen und dieses Problem auszuschließen, hat diese Forschung die Diskussion über die Hauptelemente des kollektiven Prozesses als problematisch, um zu zeigen, dass es sich um ein kohärentes und harmonisches Verfahrens System handelt. Die verwendete Methodik wird eine bibliographische Überprüfung sein. So stellt die Arbeit als Ergebnis die Systematisierung grundlegender Elemente zum Verständnis des kollektiven Schutzes dar, wenn es um die rechtlichen und doktrinären Bestimmungen zum Schutz Solidaritätsrechte geht, um das Feld für ein Verständnis des kollektiven Schutzes zu öffnen.

Schlüsselwörter: Kollektiver Prozess, Solidaritätsrechte, Zivilprozessrecht, Prinzipien.

\footnotetext{
${ }^{1}$ Master-Abschluss in Menschenrechten und Demokratie von der UFPR-Föderalen Universität von Paraná. Bachelor in Rechtswissenschaften von der UCSal-Katholischen Universität von Salvador. ORCID: https://orcid.org/0000-0001-6503-9625.
} 


\section{EINLEITUNG}

Der kollektive Schutz ist ein wesentliches Verfahren Instrument zur Verwirklichung sozialer Gerechtigkeit durch den Schutz von Rechtsgütern, die nicht nur einem Individuum gehören, sondern die Kollektivität betreffen. Es stellt sich heraus, dass die gesetzlichen Bestimmungen über das Institut in unterschiedlichen Rechtsvorschriften angesiedelt sind und nicht auf das verfassungsrechtliche Verständnis der Angelegenheit verzichten, was ein Hindernis für die kohärente Untersuchung des kollektiven Prozesses darstellen kann. Angesichts dieses Problems zielt diese Forschung darauf ab, eine Reflexion über die wesentlichen Elemente des kollektiven Schutzes Solidaritätsrechte, wie das Konzept der Solidaritätsrechte, Legitimität und Kompetenz, anzubieten. So wird der kollektive Prozess in kohärenter und harmonischer Weise mit den leitenden Verfassungsprinzipien dargestellt, um Hermetik zu beseitigen, die durch das Wissen um kollektiven Schutz wahrgenommen werden kann. Die verwendete Methodik wird die bibliographische Begutachtung von Autorinnen und Autoren sein, die einen wichtigen Beitrag zum Verständnis des Schutzes Solidaritätsrechte geleistet haben.

Aus verfassungsrechtlicher Sicht ist der Grundsatz des ordnungsgemäßen Gerichtsverfahrens (Art. 5 LIV, KF/88) in seiner kollektiven Dimension auszulegen, da er darauf abzielt, Formalitäten zu reduzieren, um ein wirksames und faires metaindividuelles Verfahren zu gewährleisten zur Gerichtsbarkeit. Das kollektive Verfahren System weist verschiedenen Entitäten Legitimität zu (ope legis), da die Relevanz der geschützten Interessen die Verantwortung verschiedener Akteure hervorruft, die Rechte eines Kollektivs zu gewährleisten. In diesem Sinne ist Art. 103, der CDC legt die Wirksamkeit erga omnes oder ultra partes für die im kollektiven Verfahren getroffenen Entscheidungen fest (BELLINETTI; SOUZA, 2019).

Was die Klassifizierung betrifft, so teilt Zavascki (2005, S. 18) die Interessen kollektiv in zwei Kategorien ein. Die erste wären homogene Einzelinteressen, die ihren kollektiven Charakter für eine empirische, kontingenz hafte, nicht notwendige Frage hätten, in der es mehrere Subjekte gäbe, aber das Objekt teilbar ist. Die zweite 
Kategorie würde diffuse und kollektive Interessen umfassen, die sich aus solchen zusammensetzen, die ihrem Wesen nach kollektiv sind und ein unteilbares Objekt und eine unbestimmte Anzahl von Subjekten betreffen. In Bezug auf Solidaritätsrechte Interessen ist es möglich, sie in zwei getrennten Bereichen zu verstehen: homogene individuelle Rechte und stricto sensu und diffuse kollektive Rechte.

Laut Ronaldo Santos (2014) besteht die Idee des Schutzes des Kollektivrechte darin, soziale Gerechtigkeit für beleidigte soziale Gruppen zu gewährleisten oder Situationen zu legalisieren, die zuvor keinen gerichtlichen Schutz hatten. Für den Autor hatte die kollektive Aktion als Vorläufer die Bill of Peace, die bis in die Geschichte des 17. Jahrhunderts zurückreicht:

[...] tratava de uma autorização para o processamento de uma ação individual sob a forma coletiva, concedida nas hipóteses em que o autor requeria que o provimento da demanda englobasse os direitos de todos os indivíduos que estivessem em igual condição de litígio, para que a questão fosse tratada de modo uniforme e com vistas a evitar a multiplicação de processos (SANTOS, 2014, p. 238).

Mit einem Schwerpunkt auf dem Verhältnis der Solidaritätsrechte als Grundrechte versteht Hachem, dass erstere die gleichen Elemente aufweisen, die mit den Grundrechten der drei Generationen zusammenhängen würden, nicht nur der dritten, zweiten oder ersten Generation:

\footnotetext{
Por conta da complexidade de sua natureza jurídica e estrutura normativa, todos os direitos fundamentais reúnem concomitantemente a totalidade dos traços que supostamente seriam peculiares a cada uma das gerações: (i) impõem deveres negativos ao Estado; (ii) dirigem ao Poder Público obrigações de fornecer prestações fáticas e normativas; (iii) ostentam a titularidade transindividual alegadamente exclusiva dos "direitos de terceira geração", bem como, simultaneamente, a titularidade individual pretensamente típica dos "direitos de primeira e segunda geração" (HACHEM, 2013, p. 621).
}

Durch kollektive Forderungen wird der Zugang zur Justiz eines unbestimmten Kollektivs oder einer bestimmten Personengruppe erhöht. Die Vorteile dieser Aktionen sind die Sozialisierung und Demokratisierung des Prozesses mit der 
Verwirklichung von Rechten und der Veränderung der sozialen Realität; die Möglichkeit, ein Delikt zu korrigieren, das aufgrund seines geringen besonderen Wertes in einer individuellen Forderung nicht postuliert würde, aber kollektiv große soziale Auswirkungen hat; Gleichberechtigung zwischen den Prozessparteien; die Molekularisierung der Forderung und Umsetzung des Grundsatzes der Rechtssicherheit und des legitimen Vertrauens angesichts einheitlicher Entscheidungen für gleichartige Fälle; die Reduzierung von Anforderungen an die Justiz, unter anderem.

Genauer gesagt tragen homogene Einzelinteressen die Kleidung individueller Interessen, aber angesichts bestimmter Gesellschafts Konstellationen besteht die Notwendigkeit, kollektiv geschützt zu werden. Die Nomenklatur selbst übersetzt bereits einen Teil des Konzepts dieses Interesses, so Zavasckis Lektion:

$\mathrm{Na}$ verdade, a própria denominação interesses individuais homogêneos já exibe dois enunciados: de um lado, trata-se de interesses ontologicamente individuais, não coletivos; de outro lado, que consistem numa multiplicidade de interesses individuais apresentando um determinado grau de uniformidade ou homogeneidade, sua característica mais importante (ZAVASCKI, 2005, p. 18).

Nach Artikel 81 des Gesetzes 8.078/91 beruhen homogene Einzelinteressen auf der Idee der gemeinsamen Herkunft, auf der schädlichen Tatsache, dass unzählige Menschen in ihrer Individualität betroffen und verletzt wurden, ohne dass ein grundlegendes Rechtsverhältnis zwischen den Inhabern oder zwischen den Parteien oder eine Einheit in Bezug auf Tatsache oder Zeitlichkeit erforderlich ist (ZAVASCKI, 2005, S. 21).

Homogene Einzelinteressen sind solche, die in ihrem Ursprung eine schädliche Tatsache haben, die eine Kollektivität beeinflusst hat und die Sphäre des individuellen Interesses überwindet. Daher haben diese Rechte einen gemeinsamen Ursprung, der sich aus einem omissiven oder kommissiven Verhalten der gegnerischen Partei ergibt, das Rechte berührt, die sich dem bloßen individuellen Aspekt entziehen und die kollektive Anmaßung schaffen, indem sie Fragen in die Diskussion einbringen, die dem singulären bloßen Deckel überlegen sind. 
Nach Zavasckis Lektion (2005, S. 21) sind sie teilbar, individuell angemessen, verfügbar, übertragbar und vom Inhaber selbst vor Gericht vertreten, episodisch solidarisch, subjektiv bestimmt und direkt reproduzierbar.

Das Merkmal der Individualität weicht jedoch nicht von dem eminenten solidarisch Gehalt homogener individueller Rechte ab. Zusammengenommen gehen diese Rechte weit über die Sphäre der besonderen Interessen ihrer Inhaber hinaus. Das kollektive Merkmal liegt nicht in der Unmöglichkeit, individuell angemessen oder ihre Inhaber nicht bestimmbar zu sein, sondern in dem Umfang, den die Verletzung der Gesellschaft offenbart (ZAVASCKI, 2005, S. 21-22). Die Verletzung homogener Einzelinteressen verletzt den sozialen Frieden und fördert ein großes soziales Unbehagen, das einen synergistischen Effekt erzeugt, da beide verstärkt werden.

Es wäre auch möglich, relevante Einzelinteressen oder gemeinsame Interessen zu benennen, was nicht getan wurde, weil Homogenität für die Möglichkeit des kollektiven Rechtsschutzes unerlässlich ist (ZAVASCKI, 2005, S. 19).

Auch in Bezug auf Solidaritätsrechte ergeben sich die Kategorien der diffusen und kollektiven Rechte im stricto sensu. Erstens sind diffuse Rechte jene Kollektivinteressen, die unteilbar sind, deren Inhaber nicht bestimmbar sind und zwischen denen kein Rechtsverhältnis besteht (DIDIER JUNIOR; ZANETI JUNIOR, 2014, S. 76-78). Der Begriff der diffusen Rechte ergibt sich aus der Analyse von Art. 81, I des Verbraucherschutzgesetzes, die als unteilbare Solidaritätsrechte Interessen begriffen werden, deren Inhaber unbestimmt und durch eine gemeinsame Tatsache verbunden sind.

Diffuse Rechte sind solche, die zu einer Kollektivität von Subjekten gehören, die unbestimmt sind, unteilbare Rechte tragen und durch eine Tatsachen Situation verbunden sind (superveniente). Das heißt, die Beziehung zwischen den Inhabern entstand zu einem Zeitpunkt nach der Verletzung, da es keinen Zusammenhang zwischen dem Verletzten zu einem früheren Zeitpunkt und dem Umstand gab, der Anlass zu kollektivem Schutz gab (DIDIER JÚNIOR; ZANETI JÚNIOR, 2014, S. 78). 
Dann gibt es die kollektiven Rechte im stricto sensu, die in Art. 81, II der CDC definiert sind. Es handelt sich um Solidaritätsrechte, mit dem Unterschied, dass es sich bei ihren Inhabern um bestimmte definierte Personengruppen handelt, d.h. es besteht ein grundlegendes Rechtsverhältnis zwischen den Inhabern, die unbestimmt, aber bestimmbar sind. Mit anderen Worten, der Unterschied zwischen diffusen und stricto sensu Kollektivrechten besteht darin, dass erstere unbestimmte Eigentumsrechte haben und letztere bestimmte kollektive Kategorien als Eigentümer haben, sodass Personen bestimmt werden können (DIDIER JÚNIOR; ZANETI JÚNIOR, 2014, S. 78-79 ).

Für stricto sensu-Kollektivinteressen ist es wichtig zu ermitteln, welcher Personenkreis geschädigt wurde, da das Recht unteilbar ist und die Inhaber einer bestimmten Kategorie sind (DIDIER JÚNIOR; ZANETI JÚNIOR, 2014, S. 78).

Es ist erwähnenswert, dass das grundlegende Rechtsverhältnis vor dem Ereignis des schädigenden Ereignisses liegen sollte. Das heißt, das Rechtsverhältnis zwischen den Inhabern ergibt sich nicht aus dem Schaden, wie es bei diffusen Rechten der Fall ist, sondern aufgrund der Einhaltung der Arbeitskategorie, des Vereins, einer bestimmten Gesellschaft und anderer Ursachen, die der Verletzung vorausgingen (DIDIER JÚNIOR; ZANETI JÚNIOR, 2014, S. 78). Auf diese Weise sind die Rechteinhaber vollständig bestimmbar und gehören zu einer Kollektivität, die individualisierbar ist.

\section{LEGITIMITÄT}

In Situationen mit singulärer Gerichtsbarkeit sind die betreffenden Themen fast immer nur für die am Kurs beteiligten Parteien von Interesse, ohne ein direktes Gewicht auf Personen zu haben, die nicht am passiven oder aktiven Pol des Kurses beteiligt sind.

Traditionell erfordert die aktive Legitimität des Schutzes individueller Interessen eine Überprüfung des Eigentums und geht auf das Erbe des römischen Rechts zurück (DIDIER JÚNIOR; ZANETI JÚNIOR, 2014, S. 79). Auf der anderen Seite ist das 
Institut des Rechts im Namen einer anderen Person nicht viel aktueller, da "[...] im Mittelalter noch nicht der Begriff der Rech tspersönlichkeit existierte, so dass es nicht einmal möglich war, davon auszugehen, dass jemand von einer anderen Person vertreten wurde; gewöhnliche Legitimität war die absolute Regel" (ZAVASCKI, 2005, S. 11).

Im brasilianischen Rechtssystem ist die allgemeine Regel in Artikel $18 \mathrm{der}$ Zivilprozessordnung vorgesehen, der das Eigentum an dem Recht als grundvoraussetzung für seine Erörterung festlegt, da die Diskussion über das Recht, das der Person eines anderen gehört, vorbehaltlich der gesetzlich ausdrücklich vorgesehenen Fälle bestehen bleibt.

Neben der gewöhnlichen Legitimität, die in Art. 18 der CPC dargestellt wird, gibt es jedoch ausnahmsweise eine außerordentliche aktive Legitimität, die in ausdrücklich gesetzlich vorgesehenen Fällen zugelassen wird, in denen sich jemand im Namen des Inhabers auf das Recht beruft. In diesem Fall gibt es eine legislative Option für Fälle, in denen es möglich sein wird, sich vor Gericht auf das Recht anderer zu berufen.

Abgesehen von den von der juristischen Person vorgesehenen Fällen müssen die Hypothesen der außerordentlichen Legitimität als logische Konsequenz des Rechtssystems betrachtet werden. Neves (2016) ist einer der Autoren, die sich zur Möglichkeit einer außergewöhnlichen Legitimität äußern, wenn keine ausdrückliche gesetzliche Erlaubnis vorliegt:

Apesar de o art. 18, caput, do Novo CPC prever que a legitimação depende de autorização expressa da lei, a melhor doutrina entende que, além da previsão legal, também se admite a legitimação extraordinária quando decorrer logicamente do sistema, como acontece com a legitimação recursal do advogado em apelar do capítulo da sentença que versa sobre seus honorários advocatícios (NEVES, 2016, p. 188).

Die Flexibilität in Bezug auf die Regel von Art. 18 der CPC scheint eine bessere Form für die Wirksamkeit des Instituts zu sein, das die Interessen des Kollektivs 
verteidigen soll. Es wird jedoch gewarnt: Die Flexibilisierung bringt nicht als unvermeidliche Folgemaßnahme ein einfacheres Verfahren mit sich.

Es fällt auf, dass das gleiche Recht Menschen aus verschiedenen sozialen Gruppen garantiert werden kann, was mit einer gewissen Häufigkeit auftritt, z. B. bei einer Verletzung sozialer Garantien. Daher ist es schwierig zu behaupten, dass es eine Legitimität gibt, alle Interessierten zu vertreten, angesichts des großen kulturellen, sozialen oder wirtschaftlichen Unterschieds, der zwischen den Rechteinhabern in kollektiven Forderungen besteht.

In diesem Zusammenhang (ZAVASCKI, 2005, S. 15) begannen die Schwierigkeiten bei der Verteidigung kollektiver Interessen. Wenn Individuen unterschiedlich sind, wie gehen Sie dann davon aus, dass einer von innen die Legitimität hat, für alle die Verteidigung dieses gemeinsamen Interesses zu fordern? Wie können die Wirkungen des kollektiven Schutzes auf diejenigen ausgedehnt werden, die nicht vor Gericht stehen, wenn nicht davon ausgegangen werden kann, was das berechtigte Interesse dieser anderen ist? Wie fordern Sie, dass große Gruppen vor Gericht erscheinen? Wie macht man das, wenn die Gruppenmitglieder noch nicht einmal bestimmt sind?

Angesichts der Unbestimmtheit der Inhaber, eines großen Unterschieds, der zwischen innen besteht, und der praktischen Schwierigkeit aller, bestimmte Fragen vor Gericht zu bringen, ist die Legitimation von Entitäten, die ihren Zweck haben, die Suche und Verteidigung der Interessen einer Kollektivität.

Auf diese Weise sind die kollektiv Legitimierten nicht Inhaber der Rechte, die legitimiert sind, sich zu berufen, und die Inhaber dieser Rechte haben das aktive Eigentum geleert (NEVES, 2016, S. 189). Par excellence könnte in Zavasckis Brief (2005, S. 15) die aktive Legitimität in kollektiven Forderungen als Repräsentation des Abwesenden bezeichnet werden.

In der kollektiven Gerichtsbarkeit gibt es keinen direkten Zusammenhang zwischen dem Eigentum an dem Recht und der Legitimität, aber es gibt eine Rechtssituation, 
die die Fähigkeit bestimmter Institutionen mit sich bringt, Interessen zu verteidigen, die nicht auf die Idee des Individualismus beschränkt sind, was die Konfiguration einer außergewöhnlichen Legitimität mit sich bringt. In diesem Sinne:

\begin{abstract}
A legitimação ao processo coletivo é extraordinária: autoriza-se um ente a defender, em juízo, situação jurídica de que é titular um grupo ou uma coletividade. Não há coincidência entre o legitimado e o titular da situação jurídica discutida (DIDIER JUNIOR; ZANETI JUNIOR, 2014, p. 21).
\end{abstract}

Das brasilianische Rechtssystem akzeptierte die Option, die Legitimations Fähigkeit bestimmter Institutionen zur Verteidigung kollektiver Forderungen anzunehmen (ZAVASCKI, 2005, S. 102). Das heißt, diejenigen, die für die Zwecke kollektiver Klagen legitimiert sind, ergeben sich aus der rechtlichen Einrichtung, wie es in Art. 5 des Gesetzes über öffentliche Zivilklagen der Fall ist, in dem die Staatsanwaltschaft, die Staatsanwaltschaft, die Staatsanwaltschaft, die Bundesvereinigung, die Länder, der Bundesbezirk, die Gemeinden und andere Subjekte als Vertreter des Kollektivs aufgeführt sind und öffentliche Zivilklagen einreichen können.

In derselben Bewegung der LACP unterstreicht Artikel 82 des Verbraucherschutzgesetzes die aktive Legitimität der Staatsanwaltschaft, der Union, der Staaten und Gemeinden, der Gemeinden, der öffentlichen Unternehmen, der Stiftungen, der gemischtwirtschaftlichen Gesellschaften oder Verbände zur Verteidigung der diffusen, kollektiven oder individuellen homogenen Rechte der Verbraucher.

Darüber hinaus wirft Zavascki (2005, S. 132) in Bezug auf die aktive Legitimität für die Zwecke der Sammelklagen einen umstrittenen Aspekt auf, der die Möglichkeit der Staatsanwaltschaft umfasse, homogene Einzelinteressen zu verteidigen, da der CRFB/88 dem parquet diese Funktion nicht verleihe.

Die Verfassung Sprache führt in artikel 129 Punkt III die Verteidigung homogener Einzelinteressen nicht als eine der Zuschreibungen der Staatsanwaltschaft auf, deren Tätigkeit mit dem Verfassungstext verknüpft ist. So ist es möglich, den Mangel 
an Entschlossenheit, diese Interessen zu verteidigen, als rechtlichen Zaun zu interpretieren (ZAVASCKI, 2005, S. 132).

Andere plädieren für die uneingeschränkte Legitimität der Staatsanwaltschaft (ZAVASCKI, 2005, S. 133). Je nach Artikel 129, Punkt IX des CRFB/88, ist es die Aufgabe des ABGEORDNETEN, Aufgaben wahrzunehmen, die mit seinem Zweck vereinbar sind, wozu auch die Verteidigung der nicht verfügbaren sozialen und individuellen Interessen im Licht von Art. 127 CRFB/88 gehört. Für diese aktuellen, solidarisches Interessen gilt eine Vermutung gesellschaftlicher Relevanz, die das parquet wirken lässt (ZAVASCKI, 2005, S. 133).

Schließlich gibt es die These der eingeschränkten Legitimität, die sich aus der Idee ergibt, dass der Staatsanwalt uneingeschränkt legitim wäre, mit der Warnung, dass Angelegenheiten von gesellschaftlicher Relevanz nur solche sein könnten, die Kongruenzen mit den verfassungsrechtlichen Funktionen des parquet enthalten (ZAVASCKI, 2005, S. 132), dies gestützt auf Art. 127, CRFB/88, ohne die Anstrengung, Relevanz anzunehmen, um der Staatsanwaltschaft Legitimität verleihen zu können.

Zur Legitimität der Staatsanwaltschaft bei der Wahrung homogener Einzelinteressen kommt Zavascki zu dem Schluss, dass die Antwort auf diese Frage in der Analyse der Rechtskostüme liege, denn im Recht liege das Konzept des Interesses der gesellschaftlichen Relevanz, das die aktive Legitimität des parquet sicherstellen werde (ZAVASCKI, 2005, S. 132). Auf diese Weise lehrt Zavascki, dass die Interessen von gesellschaftlicher Relevanz konstituiert werden durch: "Auferlegung der Nichtverfügbarkeit, die direkte Zuweisung von Legitimität an die Staatsanwaltschaft zu ihrer Verteidigung und die ausdrückliche Charakterisierung der sozialen Relevanz" (ZAVASCKI, 2005, S. 136).

Das Delinde ist unumstritten. Ohne den von Zavascki dargestellten Aspekt der gesellschaftlichen Relevanz besteht keine Notwendigkeit, über die Legitimität des parquetzu sprechen, um zur Verteidigung homogener Individueller Interessen zu handeln. 
Schließlich sind die Merkmale hervorzuheben, die Didier in der aktiven kollektiven Legitimation offenbart, wie etwa die Regulierung durch die Kunst. 5 des Bundesgesetzes Nr. 7,347/85 und Art. 6 Abs. 1 S. 1 lit. 82 der CDC, von juristischen Personen gehalten werden, die nicht in ihrem eigenen Namen handeln, da die Inhaber nicht antragsberechtigt sind:

Enfim, o certo é que a legitimação coletiva possui as seguintes características: a) está regulada, inicialmente, por lei (art. 5ํ da Lei Federal no 7.347/85; art. 82 do CDC etc.); b) é conferida a entes públicos, privados e despersonalizados, e, até, ao cidadão, na ação popular; c) o legitimado coletivo atua em nome próprio na defesa de direitos que pertencem a um agrupamento humano (pessoas indeterminadas, comunidade, coletividade ou g rupo de pessoas, titulares de direitos individuais abstratamente considerados, na forma do a1i. 81 do CDC e seus incisos); d) esse agrupamento humano não tem personalidade judiciária, portanto não pode atuar em juízo para proteger os seus direitos, 26 cuja defesa cabe aos legitimados coletivos, que possuem legitimação autônoma, exclusiva e concorrente e simples (DIDIER JUNIOR; ZANETI JUNIOR, 2014, p. 212).

\section{KOMPETENZ}

Kompetenz ist ein grundlegendes Element für die Erfüllung eines ordnungsgemäßen Verfahrens. Die Verteidigung Solidaritätsrechte erfolgt zugunsten einer Kollektivität, die getrennt werden kann und ohne jede Bindung, die sie vereint, was die Aufmerksamkeit auf den Moment der Fixierung der Kompetenz lenkt.

Für die Festlegung der territorialen Zuständigkeit in kollektiven Forderungen listet Neves sieben Schritte auf, die für die Offenlegung des zuständigen Urteils erforderlich sind. Es wird betont, dass die Fixierung der örtlichen Zuständigkeit eine Folge der Analyse der Verfahren Gesetzgebung ist, wie sie in Fällen des singulären Schutzes geschieht, da es sich um eine Angelegenheit handelt, die mit dem in isonomischer Weise behandelten Recht mit dem individuellen Schutz zusammenhängt (NEVES, 2016, S. 168).

Es muss jedoch darauf hingewiesen werden, dass die Rechtsvorschriften, die den Schutz kollektiver Rechte regeln, die Zuständigkeit bestimmter Gerichte oder 
atypischer Stellen in der Art und Weise festlegen können, wie dies bei dem besonderen kollektiven Verfahren, der Volksklage, der öffentlichen Zivilklage, der kollektiven Verfügung, dem kollektiven Haftbefehl und der Klage wegen Verwaltungs Fehlverhaltens geschehen kann.

Aufgrund der Relevanz der Maßnahmen ist es erwähnenswert, dass die Kompetenz im kollektiven Bereich von den Künsten geregelt wird. 2, das Gesetz über öffentliche Zivilklagen und 93 des Verbraucherschutz Gesetzbuchs, die als Kriterium der örtlichen Zuständigkeit den Ort des Schadens festlegen.

Darüber hinaus wäre die Zuständigkeit je nach Rechtsvorschrift des LACP territorial und funktional, was die Debatte aufwirft, ob die Zuständigkeit auf die Foren, die territoriale Frage oder die größere Wirksamkeit und Leichtigkeit aufgrund des funktionalen Kriteriums zurückzuführen wäre. Es ist jedoch sicher, dass die örtliche Zuständigkeit absolut ist, was zu ihrer obligatorischen Einhaltung führt.

Nach der Bestimmung des zuständigen Gerichtsstands beginnt die Suche nach dem zuständigen Urteil, die aus den Organisationsgesetzen der Justiz und der CPC erfolgt. Dies liegt daran, dass die Zuständigkeit des Urteils von der örtlichen Zuständigkeit berührt wird, niemals umgekehrt (NEVES, 2016, S. 186).

\section{VOLLSTRECKUNG DES URTEILS IN SAMMELKLAGEN}

Die Grundlage für die Vollstreckung eines Individuellen Urteils wird durch die Erfüllung eines Kollektiven Urteils getragen, das als Fortsetzung des Erkenntnisprozesses nach dem Zeitpunkt erfolgen muss, zu dem der Schuldner dem Strafbefehl nicht nachgekommen ist. Die Umsetzungsphase wird durch die Befolgung eines Urteils gemäss den in Art. 515 CPC vorgesehenen Umständen eingehalten.

In Bezug auf diffuse und kollektive Rechte stricto sensu wird der Schutz des materiellen Rechts durch eine kollektive Vollstreckung verwirklicht, die sich zum Wohle der Gemeinschaft entfaltet, die durch die Aspekte erfolgt, die bei der 
individuellen Vollstreckung verwendet werden, ohne Verfahrensspezialität aufgrund des kollektiven Charakters der Interessen (NEVES, 2016, S. 392-393).

Aus der Vollstreckung kann sich eine einstweilige Verfügung (Verbot der Rechtswidrigkeit) oder in natura Wiedergutmachung, wie zum Beispiel Schadenersatz, oder in bar, die in diesem Fall der geschädigten juristischen Person des öffentlichen Rechts oder dem Diffuse Rights Fund zuzufallen ist (Art. 13 des Bundesgesetzes Nr. 7.347/1985) (DIDIER JÚNIOR; ZANETI JÚNIOR, 2014, S. 412).

Was die Legitimität betrifft, so kann jeder integrale Bestandteil des aktiven Pols die Ausführung fördern, auch wenn er nicht an der Erkenntnis Phase teilgenommen hat, gemäß der Auslegung von Artikel 15 des Gesetzes 7.347, der als Anforderung die Zeitspanne von 60 Tagen des endgültigen Transits aufgelistet, damit neue Subjekte am Prozess teilnehmen können, um die Umsetzungsphase einzuleiten (DIDIER JÚNIOR; ZANETI JÚNIOR, 2014, S. 413). Mit anderen Worten, die kollektive Durchsetzung diffuser oder kollektiver Rechte stricto sensu kann von jeder außerordentlich legitimierten Person unter Berücksichtigung des zeitlichen Ablaufs der Rechtskraft durchgeführt werden. Dies ist auch in Art. 15 des CBPC-IBDP und Art. 26 des CBPC-UERJ/UNESA.

Hervorzuheben ist die Verpflichtung, die Einhaltung des Urteils als wesentliche Zuschreibung zum parquet unter Wahrung kollektiver Interessen zu dokumentieren (NEVES, 2016, S. 391).

Es ist jedoch zu unterscheiden, dass die Verpflichtung des Staatsanwalts, ein Urteil in einer öffentlichen Zivilklage zu vollstrecken, nach 60 Tagen nach der endgültigen Urteilsverkündung des Urteils gemäß Art. 15 des Gesetzes 7.347/85 entsteht.

Bei Sammelklagen ist die Staatsanwaltschaft zur Vollstreckung des Verurteilung Urteils verpflichtet, wenn sich der Bürger nach Art. 16 des Gesetzes 4717/65. Mit anderen Worten, es bestünde für Parkett in Bewegung eine funktionale Pflicht zur Einhaltung eines vorläufigen Urteils, da die Möglichkeit zur Änderung des Set Kommandos gegeben wäre. 
Daher besteht auch die Möglichkeit der Einzelvollstreckung bei der Wahrnehmung diffuser und kollektiver Rechte stricto sensu, nach Didiers Lehre:

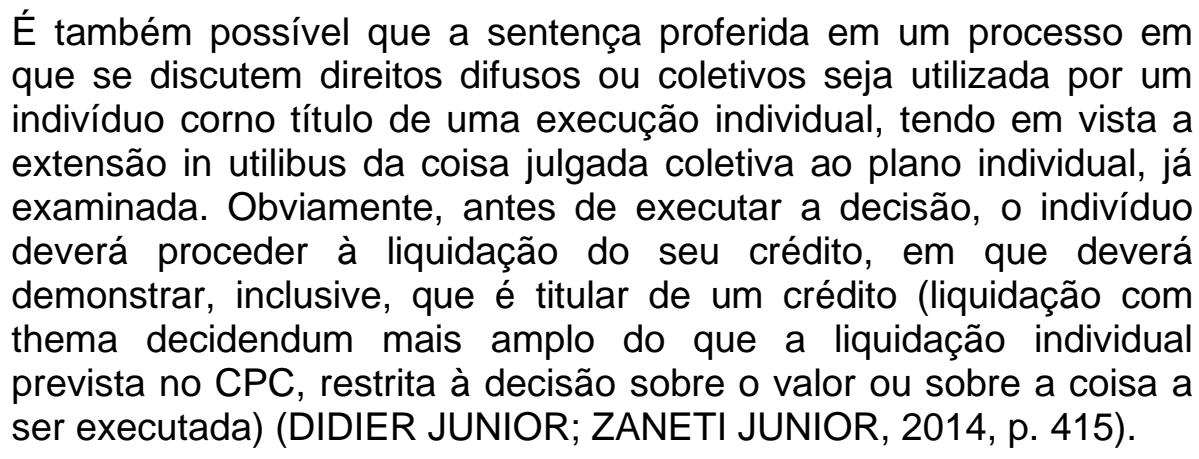

Was homogene individuelle Rechte betrifft, bei denen es sich um individuelle Rechte handelt, die nach ihrem gemeinsamen Ursprung zusammengefasst sind, so wird die Durchsetzung von den Inhabern individuell durchgeführt. Das heißt, die starke Kollektivität Komponente, die in der Wissens Phase existierte, verlangsamte sich in der Ausführungsphase, da das individuelle Interesse in der Ausführungsphase als kollektiv behandelt wird (NEVES, 2016, S. 394). Was die Foren betrifft, so schreibt Art. 516 CPC eine strenge Regel, ein Kriterium der absoluten Zuständigkeit, vor, das zur Befolgung eines Urteils für die Entscheidung führen würde, die den Fall im ersten Grad der Zuständigkeit entschieden hat.

Die Auslegung dieser Rechtsvorschrift schließt jedoch die individuelle Vollstreckung eines Sammelurteils ein, verteidigt Neves (2016, S. 376) jedoch nach Kriterien der Praktikabilität den Gerichtsstand des Wohnsitzes des Verwalters als zuständig für die individuelle Beilegung von Kollektiv Urteilen, so dass es keine große Konzentration von Verfahren in dem Bezirk gibt, der über den Fall im ersten Grad der Zuständigkeit entschieden hat, und eine geringere Belastung des Exequenten.

Es sei jedoch darauf hingewiesen, dass in Bezug auf den einzigen Absatz von Art. 97 CDC ein Veto des Präsidenten vorlag, das die Hypothese ausschließt, dass der Gerichtsstand für die Vollstreckung eines Kollektiv Urteils als derjenige des Wohnsitzes des Exequien gewählt wird. 
Nach Zavascki (2005, S. 164) gibt es keine Möglichkeit, das Veto als nutzlos zu betrachten, da es den Weg zur Interpretation öffnet und Gründe aufzeigt, die berücksichtigt werden sollten, da sie vom Gesetzgeber berücksichtigt werden.

In Anbetracht der ausdrücklichen Bestimmung der Zuständigkeit für die Vollstreckung des Urteils, des Urteils, das den Fall im ersten Grad der Zuständigkeit verhandelt hat, bestehen nach Art. 516, II CPC keine Zweifel an der Zuständigkeit.

Schließlich gibt es einen umstrittenen Punkt in Artikel 98 der CDC in Bezug auf die Möglichkeit der Staatsanwaltschaft und der öffentlichen Stellen (legitimiert durch Art. 82 der (DC), die Ausübung homogener Einzelinteressen zu fördern, da die kollektive Sphäre zum Zeitpunkt der Ausübung von Einzelinteressen leer ist. Hervorhebung von Zavasckis Lektion:

Quanto ao Ministério Público e órgãos públicos, não se vislumbra circunstância na qual tais entes teriam algum interesse em promover liquidação e execução específicas em favor de particulares, ainda mais mediante representação. Em consequência, é certo não possuir - Ministério Público legitimidade para promover o tratamento individualizado da sentença genérica, mas não porque ele não tem poderes para agir em nome próprio em favor das vítimas e sucessores e sim porque, nessa hipótese, não se trata de defesa de interesses sociais ou individuais indisponíveis (ZAVASCKI, 2005, p. 163).

Schließlich ist es interessant festzustellen, dass auch diejenigen, die nicht Teil des

Prozesses sind, sich über das beschweren können, was in einem kollektiven Urteil vorgeschlagen wurde, da sein Zweck darin besteht, den Rechten der Gemeinschaft Konkretisierung zu verleihen:

Diante disso, os instrumentos processuais utilizados para tutela dos interesses transindividuais devem ser adaptados à natureza do interesse substancial em questão, de modo a lhe proporcionar uma maior efetividade. Para tanto, algumas alterações legislativas foram feitas com a finalidade de atender esse escopo. Dentre elas menciona-se a tratativa dada à sentença coletiva, à coisa julgada e à execução de sentença coletiva. A sentença coletiva, dada sua natureza, possui um alcance diferenciado no que se refere aos indivíduos por ela albergados, podendo atingir, inclusive, aquele que não foi parte no processo, dada a extensão secundum eventum 
litis da coisa julgada coletiva ao plano individual (ALFREDO, BALEOTI, 2012, p. 435).

\section{HÜTER DER DRINGLICHKEIT IN KOLLEKTIVEN AKTIONEN}

Im Allgemeinen stellt der Schutz der Dringlichkeit bei kollektiven Klagen keine Divergenz der Argumentation in Bezug auf einzelne Klagen dar (DIDIER JÚNIOR; ZANETI JÚNIOR, 2014, S. 342), die Inzidenz von CPC bringt, wenn es die in Art. 300 festgelegten Anforderungen gibt.

Es gibt jedoch bestimmte besondere Verfahren, die diese Verfahrenstechnik vorsehen, wie z. B. Art. 12 des LACP, der Möglichkeiten bietet, frühzeitig Schutz bei der Verteidigung bestimmter Kollektivinteressen zu gewähren (DIDIER JÚNIOR; ZANETI JÚNIOR, 2014, S. 342).

Beispielhaft sieht auch Art. 4 LACP die Möglichkeit von Unterlassungs Maßnahmen mit dem Anwendungsbereich vor, Schäden an den darin beschriebenen Rechtsgütern zu verhindern. Zu Recht führt Didier zur Berichtigung der Bestimmungen dieses Artikels, da die Maßnahme zufriedenstellend wäre, da sie das durch die Provokation der Rechtskraft beantragte Recht und nicht eine Sicherungsmaßnahme gewähren würde:

\footnotetext{
Embora mencione expressamente a tutela cautelar, a redação do dispositivo não dá margem a dúvida: não se trata de tutela cautelar, mas, sim, tutela inibitória, que é satisfativa e visa exatamente obter providência judicial que impeça a prática de ato ilícito e, por consequência, a ocorrência de um dano (DIDIER JÚNIOR; ZANETI JÚNIOR, 2014, p. 343).
}

Es ist wichtig, bestimmte Hindernisse für die Gewährung von vorläufigem Schutz in Sammelklagen hervorzuheben, wie die Bestimmungen von Artikel 2 des Bundesgesetzes Nr. 8,437/1992 und § 2 von Art. 22 des Gesetzes Nr. 12,016/2009, die den Erlass einer einstweiligen Verfügung in einem Haftbefehl gegen kollektive Sicherheit oder eine öffentliche Zivilklage auf die Anhörung des gerichtlichen Vertreters der juristischen Person des öffentlichen Rechts beschränken. muss innerhalb eines Zeitraums von maximal 72 Stunden durchgeführt werden. 
Es ähnelt auch dem, was für die Erteilung einer einstweiligen Verfügung im Kollektiv Streit über den Besitz von Eigentum geschieht, die von der Erfüllung der in Art. 565 der Zivilprozessordnung festgelegten Anforderungen abhängig ist.

\section{WIEDER KORRIGIERBARKEIT IN KOLLEKTIVEN HANDLUNGEN}

Es gibt kein spezifisches rekursives Medium für den Einsatz in Sammelklagen. Es gibt außergewöhnliche Erwähnungen der Korrigierbarkeit bei der Anwendung in Sammelklagen, die eine allgemeine Anwendung des wiederkehrenden Systems der Zivilprozessordnung mit sich bringt (NEVES, 2016, S. 324)

In Bezug auf die Anfechtung einer Zwischenentscheidung listet Art. 1015 des CPC die Liste der Hypothesen auf, die der Instrumente Schädigung Spielraum eröffnen. Abhängig von der ausdrücklichen Genehmigung dieses Artikels erlauben in seinem Absatz XIII andere gesetzlich vorgesehene Fälle die Behandlung der Verletzung des Instruments, in dem Artikel 19, 1 des Gesetzes 4.717/65, mit der Bestimmung, dass es angemessen ist, das Instrument aufgrund einer Zwischenentscheidung im Rahmen einer Volksklage zu verschärfen.

Nach Neves (NEVES, 2016, S. 325) sollte die oben genannte Norm aufgrund des kollektiven Mikrosystems auf alle anderen kollektiven Klagen anwendbar sein, was die Einschaltung einer Instrumente Verletzung in jede zwischenstaatliche Entscheidung, die in einem kollektiven Prozess entsteht, ermöglichen würde.

In Bezug auf die rekursive Legitimität ist es interessant zu analysieren, dass es Ausnahmen von der Regel von 996 der CPC gibt, die die unterlegene Partei, interessierte Dritte und Staatsanwälte als legitime Vermögenswerte für die Berufungen festlegt.

Es sollte betont werden, dass Artikel 996 der CPC sich mit rekursivem Interesse und nicht mit rekursiver Legitimität befasst, da im Umgang mit einer erfolglosen Partei eine Analyse des Vorliegens vorgenommen wird, die zu einer konkreten Analyse der Entscheidung führt, die das wiederkehrende Interesse in Frage stellt (NEVES, 2016, 
S. 330). Legitimität hat nichts mit Interesse zu tun, denn es ist ein legitimer Teil, auf denjenigen zurückzugreifen, der gewinnt oder verliert, das Kriterium der Feststellung der Legitimität ist völlig unabhängig vom Inhalt der Entscheidung.

Es lohnt sich, ein wichtiges Thema hervorzuheben: Gemäß der Regel von Artikel $19 \S$ 2 des Gesetzes 4.717/65 kann jeder Bürger gegen die Urteile und Entscheidungen, die im Zuge einer Volksaktion ergangen sind, Berufung einlegen. Es stellt sich heraus, dass je nach der in Artikel 996 CPC festgelegten Regel der Bürger, der nicht den aktiven Pol des Kurses bildet, nicht als erfolgloser Partei oder Dritter passt, da das Recht auf Stellungnahme in der Volksklage diffus ist, wodurch der Begriff des dritten Beteiligten im einzigen Absatz von Art. 996 entfernt wird. CPC. So stellt der Bürger nicht die Liste der Subjekte zusammen, die rekursive Legitimität haben, sondern findet im LAP die Erlaubnis, Berufung einzulegen.

Was die rekursiven Effekte (obstinent, translativ, expansiv, substitutiv, regressiv und verzögert) betrifft, so gibt es keine Besonderheit, wenn sie im kollektiven Prozess erzeugt werden. Nur in Bezug auf die aufschiebende Wirkung ist es erforderlich, die Rechtsvorschriften für jede Maßnahme zu prüfen, um das Fehlen oder Vorhandensein jeder genannten rekursiven Wirkung zu überprüfen (NEVES, 2016, S. 332).

Hervorzuheben sind die Rechtsvorschriften über die öffentliche Zivilklage, die dem Richter eine aufschiebende Wirkung auf Rechtsmittel mit dem Umfang der Beseitigung eines irreparablen Schadens für die Parteien verleihen, abhängig von Art. 14 des Gesetzes 7.347/1985, der die Auffassung bildete, dass die im Rahmen der öffentlichen Zivilklage eingelegten Rechtsmittel nicht nur wegen ihrer bloßen Interposition (NEVES, 2016, S. 332). So muss der Rechtsbehelf, der nach Art. 1.012 der Zivilprozessordnung in der Regel aufschiebende Wirkungen hat, den in Art. 14 des LACP aufgeführten Anforderungen (Vermeidung nicht wiedergutzumachender Schäden beiseite) genügen, um zu verhindern, dass das im Rahmen der öffentlichen Zivilklage ergangene Urteil unmittelbare Wirkungen entfaltet. 


\section{ENDGÜLTIGE ÜBERLEGUNGEN}

Angesichts der Diskussion, die durch diese Arbeit geführt wird, ist festzustellen, dass der Text des CRFB/88 mit dem Schutz der kollektiven Rechte an Stärke und Wirksamkeit gewinnt, und zwar in der Art und Weise, wie eine Suche nach der Verwirklichung sozialer Garantien durchgeführt wird, die in der Verfassung Sprache vorgesehen sind und gerichtlich geltend gemacht werden können.

Ein solches Instrument ist äußerst interessant für die soziale Gerechtigkeit in einer Massengesellschaft, in der die Verletzung eines Rechts über die individuelle Sphäre hinausgehen und eine Kollektivität von Menschen erreichen kann. Aus diesem Grund wurde, um auf das anfänglich gestellte Problem zu reagieren, die verfassungsrechtliche Voreingenommenheit der Garantie Solidaritätsrechte hervorgehoben, indem den Rechtsvorschriften, die kollektive Rechte im Einklang mit der Verwirklichung des Grundsatzes der sozialen Gerechtigkeit und des ordnungsgemäßen Verfahrens gewährleisten sollen, Einzigartigkeit verliehen wurde.

Durch die Untersuchung der wesentlichen Elemente des kollektiven Schutzes wurde ein harmonisches System vorgestellt, das Kohärenz findet, um den Schutz des Rechtswohls der gesamten Gemeinschaft zu gewährleisten. So wurde das Problem der Hermetik dieses Verfahrens Systems überwunden, da es so angegangen wurde, dass seine konstitutionelle Matrix und seine rechtlichen Grundlagen diskutiert wurden. Daher wird das, was einem getrennten System ähneln könnte, weil es in verschiedenen Gesetzgebungen eine Standardisierung hat, als ein integriertes Instrument interpretiert, das sich bewegt, um Rechte zu gewährleisten, die nicht nur einer Person, sondern der Gesellschaft gehören.

\section{VERWEISE}

ALFREDO, Luciana Romaneli Rodrigues e BALEOTTI, Francisco Emílio. A sentença coletiva e a efetividade dos direitos transindividuais. Revista Jurídica Cesumar, Vol. 12, No 2, 2012. 
BELLINETTI, Luiz Fernando; SOUZA, Raíssa Fabris. Obstáculos para a efetividade da tutela de direitos transindividuais por entidades sindicais em prejuízo ao efetivo acesso à justiça. Revista Cidadania e Acesso à Justiça, Florianópolis, SC, v. 5, p. 1-17, 2019.

DIDIER JUNIOR, Fredie; ZANETI JUNIOR, Hermes. Curso de Direito Processual Civil: processo coletivo. 9. ed. Salvador: JusPodivm, 2014.

HACHEM, Daniel Wunder. A dupla titularidade (individual e transindividual) dos direitos fundamentais e econômicos, sociais, culturais e ambientais. Revista de Direitos Fundamentais e Democracia, v. 14, n. 14, Curitiba, p. 618-688, jul./dez. 2013.

NEVES, Daniel Amorim Assumpção. Manual de processo coletivo. São Paulo: Método, 2016.

SANTOS, Enoque Ribeiro dos. Processo coletivo do trabalho. 2. ed., rev., atual. e ampl. Rio de Janeiro: Forense, 2018.

ZAVASCKI, Teori Albino. Processo coletivo: tutela de direitos coletivos e tutela coletiva de direitos. Tese (Doutorado Programa de Pós-graduação em Direito) Faculdade de Direito da Universidade Federal do Rio Grande do Sul, Porto Alegre, 2005.

Eingereicht: Dezember 2021.

Genehmigt: Dezember 2021. 